\title{
Determination of the significant anthropometry dimensions for user-friendly designs of domestic furniture and appliances - Experience from a study in Malaysia
}

\author{
Siti Zawiah Md Dawal ${ }^{a}$, Zubaidah Ismail b,*, Khairi Yusuf ${ }^{a}$, Salwa Hanim Abdul-Rashid ${ }^{a}$, \\ Nurul Shahida Md Shalahim ${ }^{\mathrm{a}}$, Nur Suliani Abdullah ${ }^{\mathrm{a}}$, Nabilla Sofia Mohd Kamil ${ }^{\mathrm{a}}$ \\ a Department of Mechanical Engineering, Faculty of Engineering, University of Malaya, 50603 Kuala Lumpur, Malaysic \\ ${ }^{\mathrm{b}}$ Department of Civil Engineering, Faculty of Engineering, University of Malaya, 50603 Kuala Lumpur, Malaysia
}

\section{A R T I C L E I N F O}

Article history:

Received 2 December 2013

Received in revised form 5 September 2014

Accepted 11 September 2014

Available online 28 September 2014

\section{Keywords:}

Anthropometric

Elderly

Ergonomics

Facilities design

Household facilities

Malaysian aged

\begin{abstract}
A B S T R A C T
Future design for the elderly is undoubtedly important for their survival to live independently in this challenging world. Therefore to realize the mentioned design, an anthropometric database for Malaysian elderly population need to be developed. A total of 107 participants took part in the study which involved 61 females and 46 males with ages ranging between 55 and 70 years. An earlier anthropometric database for Malaysian elderly population has been developed. Sixty body dimensions were measured in the study. It is hoped that from this study, the database can be used as a guideline in designing household facilities for the Malaysian elderly and elderly of other countries. The design of the household facilities should be influenced by sound ergonomics dimensions in order to create a safe and healthy environment for the elderly.
\end{abstract}

2014 Elsevier Ltd. All rights reserved.

\section{Introduction}

At present, life span for people all over the world are longer compared to previous decades due to improvement in the medical technologies and efficient health care system [1]. The definition of 'elderly' or 'senior citizen' according to the United Nations World Assembly on Ageing used '60 years and over' as the cut-off in deliberating ageing trends which has been adopted by Malaysian policy makers in planning for senior citizens. In Malaysia, the statistics of the elderly citizens in 2009 is about 2.03 million, which represent $7.1 \%$ of the total population and the percentage is predicted to rise to $9.8 \%$ in 2020 [2,3]. Additionally, according to the Ageing Workforce ${ }^{\mathrm{TM}} 2006$ Report [4], Asia Pacific will be home to most of the world's elderly

\footnotetext{
* Corresponding author. Tel.: +60 379675284; fax: +60 379675318.

E-mail address: zubaidah_jka@yahoo.com (Z. Ismail).
}

people with 998 million people aged 60 and above by 2050 especially in Asia's most develop countries. For example, the proportion of the population over age 50 in Singapore is set to increase from $23 \%$ to $50 \%$ over the next 25 years.

This trend is similar to the US population where the percentages of the elderly citizens depute $13 \%$ of the entire population in 2010 and is believed to increase to almost $23 \%$ by June 2050 as reported by Intergenerational Report, 2010. The same trend of higher growth in most developing world is a result of successful efforts to lower fertility levels and increase longevity [3]. This phenomenon is also influenced by the transformation in socioeconomics. In order to maintain a high standard of living, elderly people are being made to be economically useful. In addition, the practices of healthy lifestyle among the elderly today, for instance, balanced diet, exercise regularly, fitness programs and so on contribute in making them to remain 
physically fit to continue in services which require less physical strength.

The increasing number of older people in the population points to the need for concerted research efforts within the area of human factor design and aging. The elderly needs an environment and facilities to support their needs within their limitations and abilities to be independently performing their regular everyday life chores comfortably and safely [5-12]. Thus due to the above reasons, it is important to develop a significant anthropometric database to realize the future facilities design for the elderly. This study was conducted among the Malaysian population. However, the data collected and the findings can are also useful for other countries.

\section{Material and methods}

\subsection{Participants}

One-hundred-and-seven participants took part in the study which involved 61 female and 46 male. All participants were in good health and have no problem with normal mobility.

\subsection{Measuring apparatus}

Standard professional anthropometer, sliding caliper, spreading caliper and a measuring tape were used in the data collection sessions.

\subsection{Selection of body dimensions}

The selection of the anthropometry body dimensions was considered according to their significance and usefulness for the development of designing the elderly housing facilities and safety. It includes 61 body dimensions and the dimensions were made according to Pheasant [13]. To facilitate the measurements, participants were requested to wear light clothing. Table 1 presents the list of body dimensions taken and Fig. 1 illustrates the body diagrams.

\subsection{Statistical analysis}

The 5th percentile, 95th percentile, mean (M), standard deviation (SD) and coefficient of variation (CV) of the elderly anthropometric dimensions were calculated. According to Pheasant [13] the 5th percentile is essential in determining the reachability and limitation for household facilities. The 95th percentile is used to ensure adequate clearance to avoid unwanted contact or trapping. The coefficient of variance (CV) measures the precision of the participants' anthropometry dimensions taken during the measurement.

\section{Results and discussion}

Table 2 presents the anthropometric dimensions for male and female elderly aged above 55 years. In this study, it is noted that there are several measurements that cannot be measured accurately due to physical weakness and disability to perform in certain required postures. Consequently, there are few standard deviations for the anthropometry dimensions which are considerably large e.g. the arm reach upward for male which is 34.11 and for female which is $22.57 \mathrm{~cm}$.

\subsection{Facilities design}

In order to design the household facilities for the elderly the 5th and 95th percentile value of the anthropometry dimensions were selected. The continuous growth of the number of the aged people has created a big market of health care and living products [14]. Due to that, anthropometry dimensions obtained in this research study are important in order to provide the older Malaysian people to live independently. One of the most important aspects of design is that they have one or more illnesses and have movement or function difficulties [15]. Many household facilities can be designed to ease the elder people in their daily activities such as the washing machine facility, toilet and shower tools, stove, cooking utensils and facility and also storage placement [16] which could create comfort and satisfaction to the living environment [17].

Most of the daily activities require a standing posture and a study stature is one of the most important anthropo-

Table 1

Significant anthropometric body dimensions of Malaysian elderly.

\begin{tabular}{|c|c|c|c|c|}
\hline \multicolumn{5}{|l|}{ Anthropometric dimension } \\
\hline 1. Weight & 14. Back waist length & 27. Sitting shoulder height & 40. Waist circumference & 53. Face breadth \\
\hline 2. Stature & 15. Sleeve inseam & 28. Sitting elbow height & 41. Hip circumference & 54. Hand length \\
\hline 3. Standing eye height & 16. Shoulder breadth & 29. Knee height & 42. Crotch thigh circumference & 55. Palm length \\
\hline 4. Standing shoulder height & 17. Hip breadth, sitting & 30. Popliteal height & 43. Lower thigh circumference & 56. Hand breadth \\
\hline 5. Standing elbow height & 18. Forearm-hand length & 31. Head circumference & 44. Calf circumference & 57. Foot length \\
\hline 6. Waist height & 19. Buttock-knee length & 32. Neck circumference & 45. Ankle circumference & 58. Instep length \\
\hline 7. Crotch height & 20. Buttock-popliteal length & 33. Shoulder circumference & 46. Ball Foot circumference & 59. Foot breadth \\
\hline 8. Kneecap height & 21. Shoulder-elbow length & 34. Upper arm circumference & 47. Heel-ankle circumference & 60 . Heel breadth \\
\hline 9. Coat height, standing & 22. Thigh clearance & 35. Wrist circumference & 48. Head length & 61. Foot height \\
\hline 10. Span & 23. Arm reach forward & 36. Thumb circumference & 49. Head breadth & \\
\hline 11. Elbow span & 24. Arm reach upward & 37. Index finger circumference & 50. Head height & \\
\hline 12. Hip breadth, standing & 25. Sitting height & 38. Middle finger circumference & 51. Face length & \\
\hline 13. Interscye breadth & 26. Sitting eye height & 39. Chest circumference & 52. Interpupillary distance & \\
\hline
\end{tabular}



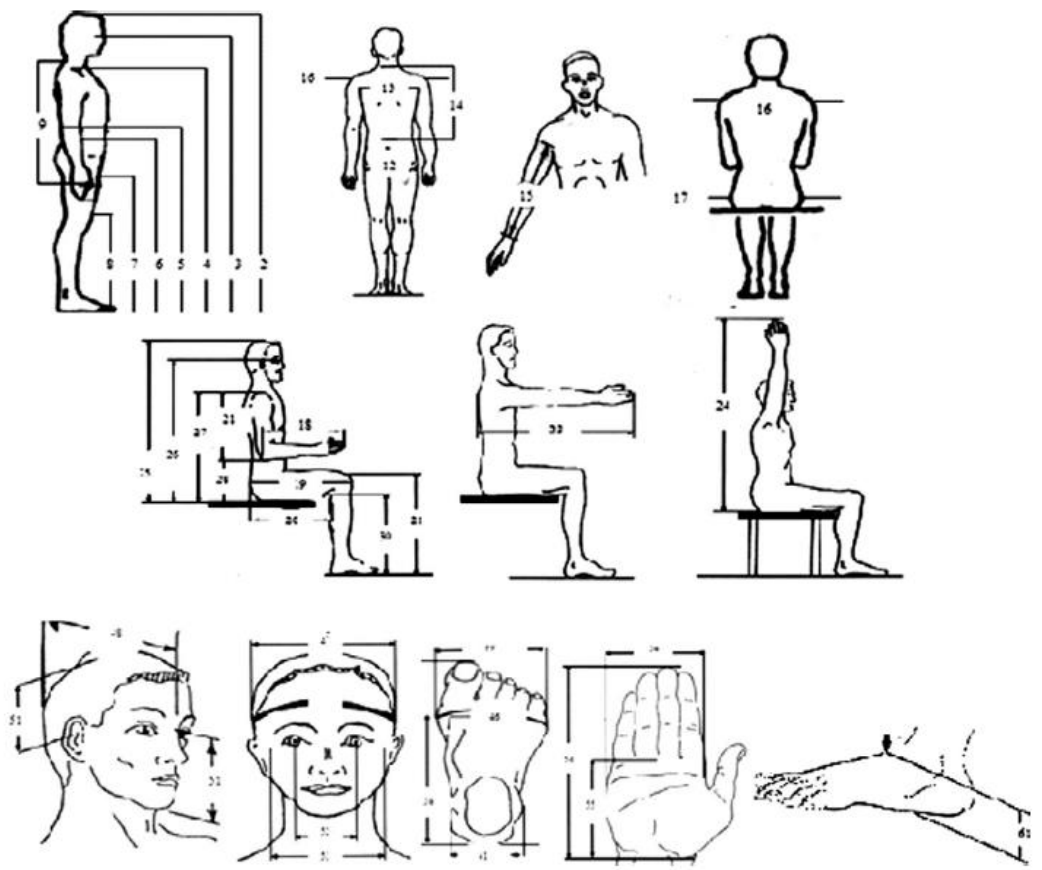

Fig. 1. Anthropometric dimensions Source: Pheasant [13].

metric characteristics affected by ageing [1]. According to Ranzijn [18] the extent of personal accommodation to the environment determines the quality of life of the older people. In line with that most of the items should be stored between the knuckle and shoulder height so that elderly users are not subjected to bending and over-reaching demands $[19,20]$.

Due to that, with the aid of new technology safe household facilities such as storage shelf, door, lavatory dimensions including hand basin and water faucet should be designed. In the kitchen or in the toilet, shower facility and switch can be designed to meet the safe and comfortable home environment for the elderly. Table 3 shows the list of the set values used for designing household facilities for the elderly. The dimension values stated in Table 3 are based on the appropriate percentiles. These chosen values will produce household features which are inclusive of the smallest to the largest dimensions of the studied population. While for standing arm reach upward, the dimension value is taken from Rashid et al. [4]. Table 4 shows the implication of standing anthropometry dimensions with the design.

The recommended dimension is the reference optimum dimension for the elderly household design to provide a safe and independent environment. Besides, it helps to promote a good posture to avoid musculoskeletal disorder among the elderly. In addition, the elderly use a minimum ability in muscular strength and joint mobility due to the ageing process. In line with that the elderly may have problems in reaching such as for shelf storage. Table 5 presents the recommended anthropometry dimensions for shelf design implicating reachability.

\subsection{Sitting anthropometry dimension implication to the elderly household}

Older people usually remain seated for a large amount of time in their accommodation every day. Static posture and prolonged sitting in a forward bending position, as elderly often acquire, puts an extreme physiological strain on the muscles, the ligaments and in particular on the discs [14]. Table 6 presents the recommended dimensions for the sitting anthropometry dimension implication.

The sitting anthropometry dimensions could also be used in designing a computer workstation for the elderly. The development provides a proper working in home environment for the elderly. All considerations will concern with the reduction or elimination of physical disorders associated with poor ergonomics design known as Musculoskeletal Stress Disorders (MSD's), which result in eye, neck and back strain, fatigue, headache, wrist, hand, elbow and shoulder diseases and Carpal Tunnel Syndrome [20]. A good posture in computer workstation helps overcome the musculoskeletal disorder problem especially for the elderly. Table 7 presents the recommended dimensions for the computer workstation for elderly. 
Table 2

Anthropometric dimensions of Malaysian elderly age 55 years and above.

\begin{tabular}{|c|c|c|c|c|c|c|c|c|c|c|c|}
\hline \multicolumn{2}{|c|}{ Dimension } & \multicolumn{5}{|c|}{ Male $(N=46)$} & \multicolumn{5}{|c|}{ Female $(N=61)$} \\
\hline & & \multirow{2}{*}{$\begin{array}{l}\mathrm{M} \\
75.05\end{array}$} & \multirow{2}{*}{$\begin{array}{l}\text { SD } \\
15.13\end{array}$} & \multirow{2}{*}{$\begin{array}{r}\text { CV (\%) } \\
20.16\end{array}$} & \multirow{2}{*}{$\begin{array}{l}5 \text { th } \\
52.50\end{array}$} & \multirow{2}{*}{$\begin{array}{l}\text { 95th } \\
99.50\end{array}$} & \multirow{2}{*}{$\begin{array}{l}\mathrm{M} \\
62.10\end{array}$} & \multirow{2}{*}{$\begin{array}{l}\text { SD } \\
14.71\end{array}$} & \multirow{2}{*}{$\begin{array}{r}\text { CV (\%) } \\
23.69\end{array}$} & \multirow{2}{*}{$\begin{array}{l}5 \text { th } \\
32.71\end{array}$} & 95th \\
\hline 1 & Weight & & & & & & & & & & 85.20 \\
\hline 2 & Stature & 157.8 & 8.60 & 5.45 & 144.6 & 169.95 & 145.4 & 18.91 & 13.00 & 134.97 & 160.39 \\
\hline 3 & Standing eye height & 146.2 & 8.75 & 5.98 & 131.4 & 158.27 & 135.6 & 7.82 & 5.77 & 122.98 & 147.22 \\
\hline 4 & Standing shoulder height & 131.8 & 7.76 & 5.88 & 117.1 & 142.15 & 119.5 & 15.40 & 12.89 & 109.62 & 130.37 \\
\hline 5 & Standing elbow height & 100.2 & 5.91 & 5.90 & 90.28 & 108.36 & 94.27 & 13.86 & 14.70 & 83.00 & 104.15 \\
\hline 6 & Waist height & 92.37 & 4.74 & 5.13 & 84.16 & 98.26 & 87.79 & 6.79 & 7.73 & 78.00 & 97.80 \\
\hline 7 & Crotch height & 71.29 & 4.94 & 6.93 & 62.48 & 78.37 & 68.12 & 6.15 & 9.03 & 57.50 & 78.01 \\
\hline 8 & Kneecap height & 43.59 & 3.19 & 7.32 & 39.02 & 49.86 & 43.49 & 6.41 & 14.74 & 35.29 & 53.43 \\
\hline 9 & Coat height, standing & 67.12 & 7.56 & 11.26 & 57.98 & 81.11 & 61.01 & 5.38 & 8.82 & 51.99 & 71.61 \\
\hline 10 & Span & 159.6 & 18.16 & 11.38 & 142.5 & 177.73 & 147.3 & 13.61 & 9.24 & 129.63 & 163.58 \\
\hline 11 & Elbow span & 83.24 & 6.67 & 8.01 & 74.81 & 91.60 & 77.10 & 13.91 & 18.04 & 62.26 & 89.51 \\
\hline 12 & Hip breadth, standing & 32.78 & 2.59 & 7.90 & 29.18 & 37.44 & 32.63 & 3.55 & 10.88 & 27.41 & 38.22 \\
\hline 13 & Interscye breadth & 31.34 & 3.38 & 10.78 & 27.40 & 36.31 & 28.64 & 2.73 & 9.53 & 23.97 & 32.24 \\
\hline 14 & Back waist length & 46.22 & 4.42 & 9.56 & 38.38 & 53.88 & 40.33 & 3.90 & 9.67 & 34.49 & 47.35 \\
\hline 15 & Sleeve inseam & 50.25 & 4.52 & 9.00 & 40.84 & 55.89 & 46.29 & 4.43 & 9.57 & 38.35 & 52.41 \\
\hline 16 & Shoulder breadth & 42.29 & 4.45 & 10.52 & 35.18 & 49.14 & 39.76 & 4.08 & 10.26 & 31.98 & 45.01 \\
\hline 17 & Hip breadth, sitting & 37.15 & 6.35 & 17.09 & 29.06 & 51.93 & 36.59 & 5.24 & 14.32 & 29.70 & 47.00 \\
\hline 18 & Forearm-hand length & 44.70 & 2.82 & 6.31 & 40.35 & 49.02 & 41.95 & 2.70 & 6.44 & 36.50 & 45.13 \\
\hline 19 & Buttock-knee length & 53.01 & 3.30 & 6.23 & 47.17 & 57.47 & 50.68 & 5.24 & 10.34 & 40.40 & 56.03 \\
\hline 20 & Buttock-popliteal length & 43.24 & 3.40 & 7.86 & 37.06 & 49.00 & 41.90 & 4.03 & 9.62 & 36.60 & 48.13 \\
\hline 21 & Shoulder-elbow length & 34.66 & 2.44 & 7.04 & 30.59 & 37.49 & 32.21 & 3.14 & 9.75 & 28.60 & 36.21 \\
\hline 22 & Thigh clearance & 12.05 & 2.25 & 18.67 & 9.19 & 16.61 & 12.07 & 3.89 & 32.23 & 8.00 & 17.4 \\
\hline 23 & Arm reach forward & 78.59 & 8.30 & 10.56 & 71.15 & 85.69 & 73.44 & 11.48 & 15.63 & 66.00 & 80.00 \\
\hline 24 & Arm reach upward & 111.9 & 34.11 & 30.48 & 39.48 & 131.75 & 105.1 & 22.57 & 21.46 & 39.10 & 121.03 \\
\hline 25 & Sitting height & 88.79 & 12.15 & 13.68 & 77.59 & 117.91 & 79.20 & 10.05 & 12.69 & 68.80 & 105.43 \\
\hline 26 & Sitting eye height & 72.05 & 8.07 & 11.20 & 60.16 & 81.10 & 66.62 & 6.01 & 9.02 & 58.00 & 79.04 \\
\hline 27 & Sitting shoulder height & 62.29 & 14.44 & 23.18 & 52.08 & 78.85 & 56.67 & 13.33 & 23.52 & 45.48 & 94.23 \\
\hline 28 & Sitting elbow height & 32.25 & 15.10 & 46.82 & 19.50 & 63.02 & 32.62 & 17.14 & 52.54 & 19.20 & 69.54 \\
\hline 29 & Knee height & 46.83 & 10.52 & 22.46 & 21.71 & 54.39 & 46.68 & 7.74 & 16.58 & 25.30 & 52.34 \\
\hline 30 & Popliteal height & 44.27 & 3.89 & 8.79 & 40.00 & 52.82 & 42.18 & 3.64 & 8.63 & 38.20 & 48.80 \\
\hline 31 & Head circumference & 55.09 & 1.44 & 2.61 & 53.05 & 57.00 & 55.16 & 2.57 & 4.66 & 51.00 & 59.36 \\
\hline 32 & Neck circumference & 40.19 & 5.09 & 12.66 & 33.10 & 47.9 & 37.31 & 5.19 & 13.91 & 30.03 & 46.9 \\
\hline 33 & Shoulder circumference & 107.9 & 8.85 & 8.20 & 96.03 & 120.00 & 99.03 & 8.53 & 8.61 & 84.10 & 114.28 \\
\hline 34 & Upper arm circumference & 30.51 & 4.60 & 15.08 & 24.00 & 38.80 & 30.45 & 5.79 & 19.01 & 22.03 & 39.95 \\
\hline 35 & Wrist circumference & 16.83 & 1.49 & 8.85 & 15.00 & 19.27 & 15.75 & 1.56 & 9.90 & 13.51 & 18.00 \\
\hline 36 & Thumb circumference & 6.51 & 4.92 & 75.58 & 5.50 & 7.50 & 6.20 & 0.51 & 8.23 & 5.50 & 7.00 \\
\hline 37 & Index finger circumference & 5.85 & 0.69 & 11.79 & 5.00 & 6.80 & 5.53 & 0.68 & 12.30 & 4.50 & 6.79 \\
\hline 38 & Middle finger circumference & 5.91 & 0.61 & 10.32 & 5.00 & 6.70 & 5.49 & 0.63 & 11.48 & 4.50 & 6.50 \\
\hline 39 & Chest Circumference & 92.05 & 16.07 & 17.46 & 79.25 & 111.18 & 93.20 & 15.9 & 17.06 & 43.99 & 111.90 \\
\hline 40 & Waist circumference & 93.66 & 13.34 & 14.24 & 72.65 & 115.62 & 92.16 & 12.84 & 13.93 & 67.62 & 106.99 \\
\hline 41 & Hip circumference & 100.8 & 12.2 & 12.10 & 83.10 & 123.58 & 100.8 & 11.89 & 11.79 & 72.81 & 126.09 \\
\hline 42 & Crotch thigh circumference & 52.49 & 7.92 & 15.09 & 41.03 & 64.00 & 50.84 & 7.69 & 15.13 & 40.02 & 61.95 \\
\hline 43 & Lower thigh circumference & 40.95 & 6.63 & 16.19 & 31.82 & 53.60 & 41.38 & 6.85 & 16.55 & 33.00 & 52.00 \\
\hline 44 & Calf circumference & 33.84 & 4.39 & 12.97 & 27.50 & 40.00 & 32.77 & 4.87 & 14.86 & 26.03 & 40.71 \\
\hline 45 & Ankle circumference & 28.23 & 31.13 & 110.2 & 19.10 & 27.95 & 22.05 & 2.59 & 11.75 & 18.72 & 26.00 \\
\hline 46 & Ball foot circumference & 24.4 & 2.01 & 8.24 & 21.55 & 27.00 & 22.51 & 2.44 & 10.84 & 17.61 & 27.68 \\
\hline 47 & Heel-ankle circumference & 31.24 & 3.10 & 9.92 & 26.55 & 35.95 & 29.25 & 2.46 & 8.41 & 25.52 & 33.00 \\
\hline 48 & Head length & 17.34 & 0.77 & 4.44 & 16.20 & 18.50 & 17.04 & 0.84 & 4.93 & 15.81 & 18.30 \\
\hline 49 & Head breadth & 15.07 & 0.84 & 5.57 & 13.58 & 16.28 & 16.28 & 12.77 & 78.44 & 13.60 & 15.60 \\
\hline 50 & Head height & 14.38 & 1.30 & 9.04 & 12.40 & 16.20 & 13.47 & 1.32 & 9.80 & 10.84 & 15.29 \\
\hline 51 & Face length & 11.72 & 1.26 & 10.75 & 9.78 & 13.48 & 12.00 & 10.8 & 90.00 & 8.31 & 13.00 \\
\hline 52 & Interpupillary distance & 6.71 & 2.15 & 32.04 & 5.00 & 10.65 & 6.24 & 0.70 & 11.22 & 5.01 & 7.49 \\
\hline 53 & Face breadth & 12.79 & 1.50 & 11.73 & 10.70 & 14.58 & 11.85 & 1.43 & 12.07 & 9.71 & 13.60 \\
\hline 54 & Hand length & 17.02 & 2.21 & 12.98 & 12.85 & 19.35 & 16.30 & 1.17 & 7.18 & 15.11 & 17.70 \\
\hline 55 & Palm length & 10.19 & 2.28 & 22.37 & 8.93 & 11.18 & 9.16 & 0.60 & 6.55 & 8.11 & 10.00 \\
\hline 56 & Hand breadth & 7.99 & 0.73 & 9.14 & 7.03 & 8.75 & 7.28 & 0.48 & 6.59 & 6.70 & 7.80 \\
\hline 57 & Foot length & 23.74 & 1.29 & 5.43 & 21.70 & 25.80 & 21.66 & 2.85 & 13.16 & 19.52 & 24.10 \\
\hline 58 & Instep length & 16.89 & 2.21 & 13.08 & 13.48 & 19.90 & 16.29 & 1.47 & 9.02 & 13.33 & 18.80 \\
\hline 59 & Foot breadth & 9.48 & 0.86 & 9.07 & 8.05 & 10.95 & 10.10 & 10.17 & 100.69 & 7.70 & 10.30 \\
\hline 60 & Heel breadth & 6.13 & 0.55 & 8.97 & 5.18 & 7.00 & 5.81 & 1.47 & 25.30 & 4.50 & 6.99 \\
\hline 61 & Foot height & 6.92 & 1.57 & 22.69 & 5.04 & 9.96 & 6.62 & 1.98 & 29.91 & 4.80 & 9.98 \\
\hline
\end{tabular}

Note: All dimensions are in $\mathrm{cm}$. 
Table 3

Body dimension values used for household design purposes.

\begin{tabular}{llr}
\hline Body dimensions & Male/female percentile & Dimension $(\mathrm{cm})$ \\
\hline Stature & Minimum, female 5th & 134.97 \\
& Maximum, Male 95th & 169.95 \\
Eye height, standing & Female 5th & 122.98 \\
Shoulder height, standing & Female 50th & 119.51 \\
Elbow height, standing & Female 5th & 83.00 \\
Waist height, standing & Female 5th & 78.00 \\
Span & Male 95th & 177.73 \\
Arm reach upward, standing & Female 5th & 149.88 \\
Arm reach forward, standing & Female 5th & 6.60 \\
Sitting height & Male 95th & 117.91 \\
Shoulder height, sitting & Female 50th & 56.67 \\
Shoulder elbow length sitting & Female 50th & 32.21 \\
Elbow height, sitting & Female 5th & 19.20 \\
Popliteal height sitting & Female 5th & 38.20 \\
Hip breadth, sitting & Female 95th & 47.00 \\
Arm reach upward sitting & Female 5th & 39.10 \\
Arm reach forward, sitting & Female 5th & 6.60 \\
Shoulder breadth & Male 95th & 49.14 \\
Elbow span & Male 95th & 91.60 \\
Forearm hand length & Female 5th & 36.50 \\
Buttock-popliteal length & Male 95th & 49.00 \\
\hline
\end{tabular}

Table 4

Standing anthropometry dimension implication for elderly household.

\begin{tabular}{lll}
\hline Type of household & Design criteria & $\begin{array}{l}\text { Recommended } \\
\text { dimension }(\mathrm{cm})\end{array}$ \\
\hline Storage shelf & $\begin{array}{l}\text { Shoulder height from floor, 5th percentile female with a } 20^{\circ} \text { (maximum) joint } \\
\text { flexion }\end{array}$ & 117.19 \\
Storage shelf (visual required) & Standing eye height from floor, 5th percentile female & 131.48 \\
Door height & Stature, 95th percentile male (minimum) & 160.39 \\
Door knob height & Standing elbow height, 5th percentile female & 90.28 \\
Peephole height & Standing eye height, 50th percentile female & 146.26 \\
Hand basin height & $10 \mathrm{~cm}$ below standing elbow height, 5th percentile female & 80.28 \\
Water faucet & $5 \mathrm{~cm}$ below standing elbow height, 5th percentile female & 85.28 \\
Shower head & Standing stature, 95th male, 10 cm clearance & 170.39 \\
Shower knob & In line with shower head, standing elbow, height 5th percentile female & 90.28 \\
Switches & Standing shoulder height of 5th percentile female & 117.19 \\
Vertical handrail (standing aid) & Elbow height (minimum), 5th percentile male Shoulder height (maximum), & Minimum = 90.28 \\
& 95th percentile male & maximum = 130.37 \\
\hline
\end{tabular}

Table 5

Standing anthropometry dimension implication for the elderly household.

\begin{tabular}{lll}
\hline Type of household & Design criteria & Recommended dimension (cm) \\
\hline Optimal height surface of shelf & Standing Upward reach, 50th percentile & 111.9 \\
\hline
\end{tabular}

Table 6

Sitting anthropometry dimension implication for the elderly household.

\begin{tabular}{lll}
\hline Type of household & Design criteria & Recommended dimension $(\mathrm{cm})$ \\
\hline Chair, Toilet bowl chair & Sitting popliteal height, 5th percentile male & 40 \\
Toilet flush placement & Sitting shoulder, 5th percentile female & 52.08 \\
Table & Sitting elbow height, 95th percentile female & 49.5 \\
Bed & Sitting height, 5th percentile female & 77.59 \\
Horizontal handrails (walking aid) & Sitting elbow height, 5th percentile female & 19.5 \\
\hline
\end{tabular}


Table 7

Anthropometry dimension implication in computer workstation.

\begin{tabular}{|c|c|c|}
\hline Type of household & Design criteria & $\begin{array}{l}\text { Recommended } \\
\text { dimension } \\
(\mathrm{cm})\end{array}$ \\
\hline Table height & $\begin{array}{l}\text { Sitting elbow height, } 95 \text { th } \\
\text { percentile female }\end{array}$ & 49.5 \\
\hline Chair height & $\begin{array}{l}\text { Popliteal height, 5th } \\
\text { percentile female (seat } \\
\text { slightly higher to allow } \\
\text { elderly to stand up and sit } \\
\text { down unassisted) }\end{array}$ & 40 \\
\hline Seat depth & $\begin{array}{l}\text { Buttock-popliteal length, } \\
\text { 5th percentile female }\end{array}$ & 37.06 \\
\hline Seat width & $\begin{array}{l}\text { Hip breadth, } 95 \text { th } \\
\text { percentile female }\end{array}$ & 37.44 \\
\hline $\begin{array}{l}\text { Back-rest height } \\
\text { above the seat }\end{array}$ & $\begin{array}{l}\text { Shoulder height, 95th } \\
\text { percentile female }\end{array}$ & 78.85 \\
\hline $\begin{array}{l}\text { Arm-rest height } \\
\text { above the seat }\end{array}$ & $\begin{array}{l}\text { Sitting elbow height, } 5 \text { th } \\
\text { percentile female }\end{array}$ & 19.5 \\
\hline Keyboard height & $\begin{array}{l}\text { Sitting elbow height, } 5 \text { th } \\
\text { percentile female }\end{array}$ & 19.5 \\
\hline Monitor height & $\begin{array}{l}\text { Sitting eye height, 50th } \\
\text { percentile female }\end{array}$ & 72.05 \\
\hline
\end{tabular}

3.3. Recommended guidelines for designing basic fixtures in the households of older Malaysian

The need to design home that fits for the elderly based on the anthropometry is important. This fact relies on several reasons, for instance, the designed home enables ageing people to better adapt to their environment, and provides an integrated strategy for well-being and satisfaction when ageing [15]. Another significant reason is to enable them to live independently in their own homes. Apart from cognitive aspect, the physical independence is also crucial, so they need to consistently concern about their health. However, unintentional injuries are the uncontrollable events. Among older people, falls are the most common accidental injuries which occur at home. Several studies have concluded that the most common sites for falls to occur are at the bathrooms, lavatories, and kitchens $[2,8]$. These findings are concurrent to the studies by other researchers $[1,4,10,15]$ which showed that older people spent most of their times at these particular areas for their activity of daily lives (ADL). With regard to the mentioned problem, this study proposed the guidelines
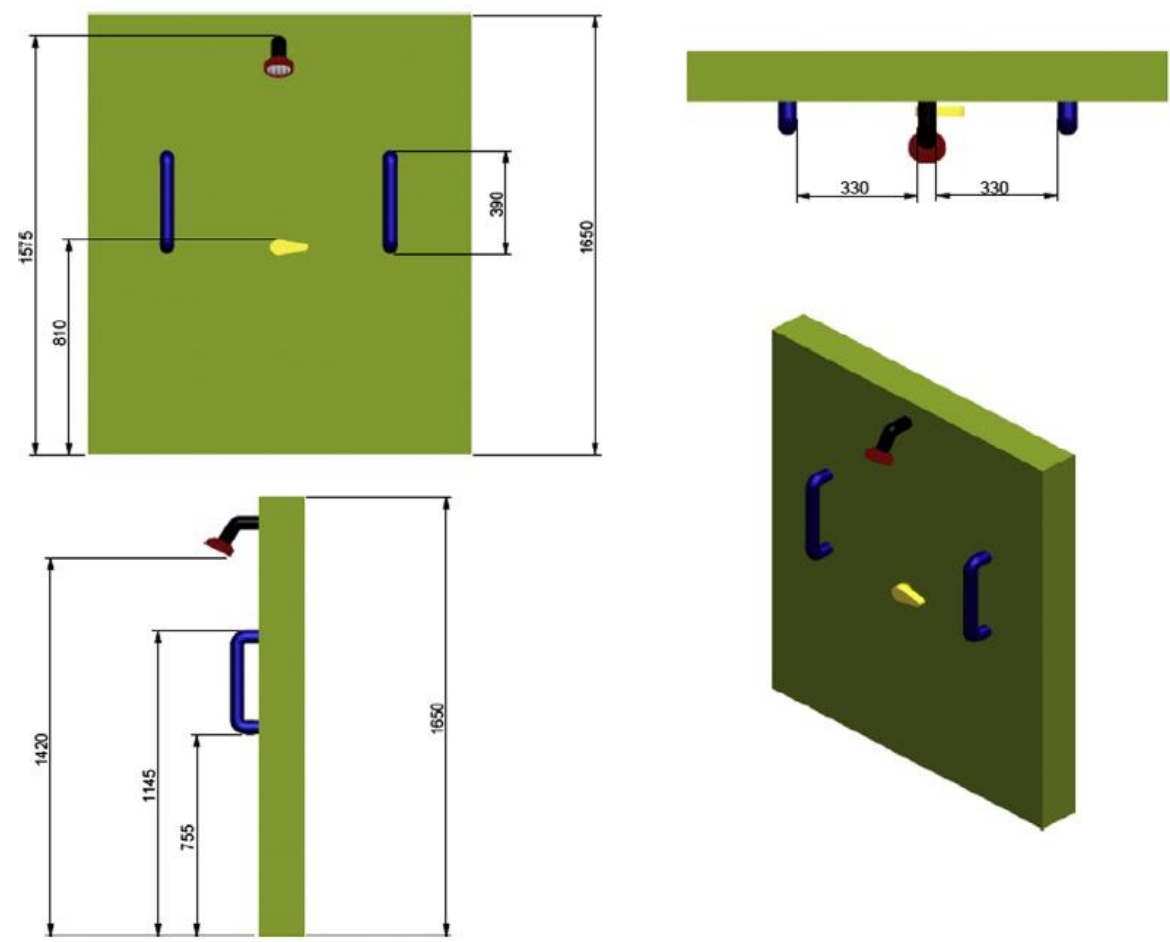

Fig. 2. 5th percentiles female (all in $\mathrm{mm}$ ). 

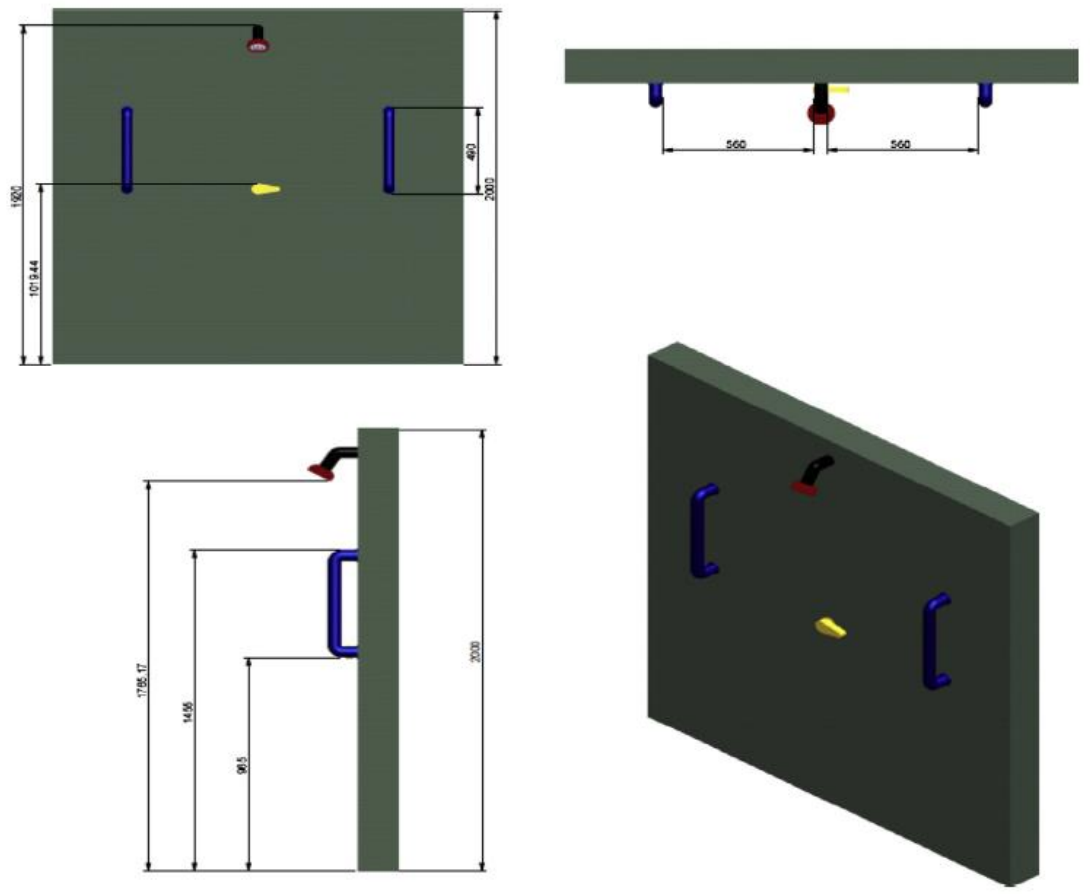

Fig. 3. 95th percale male (all in $\mathrm{mm}$ ).
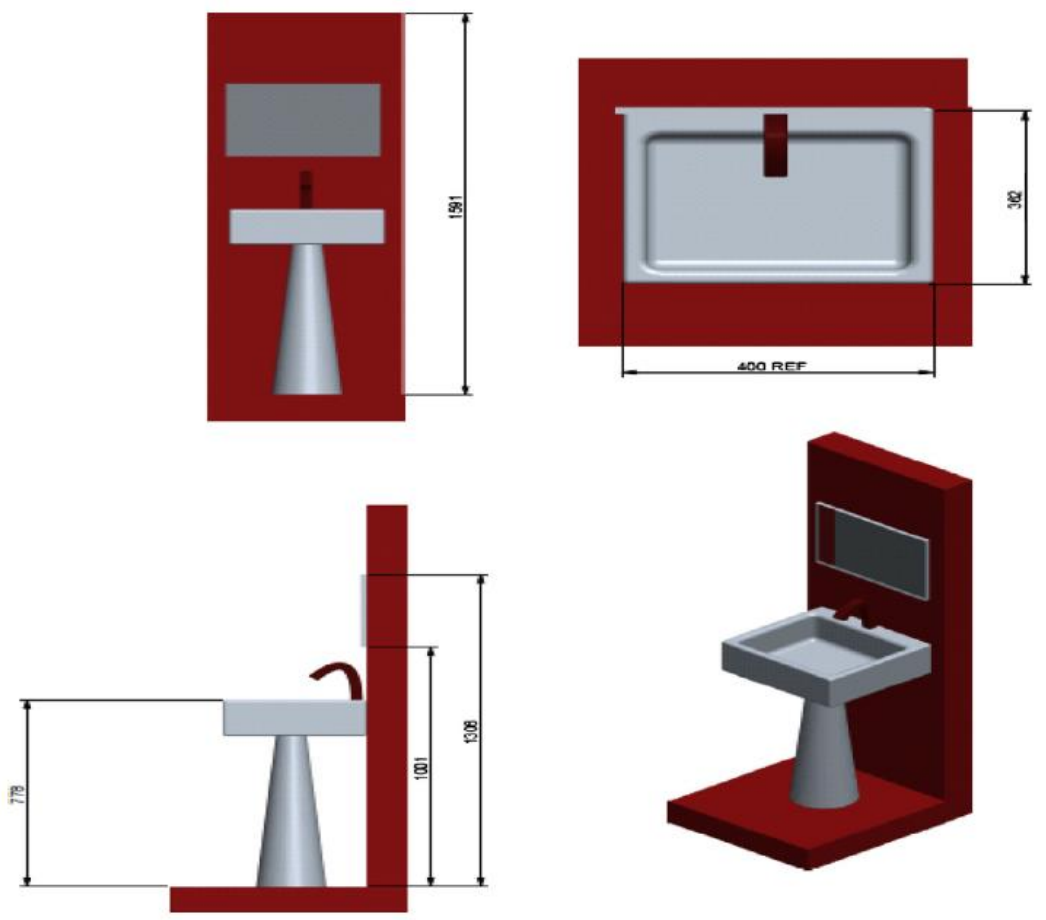

Fig. 4. 5th percentiles female (all in $\mathrm{mm}$ ). 

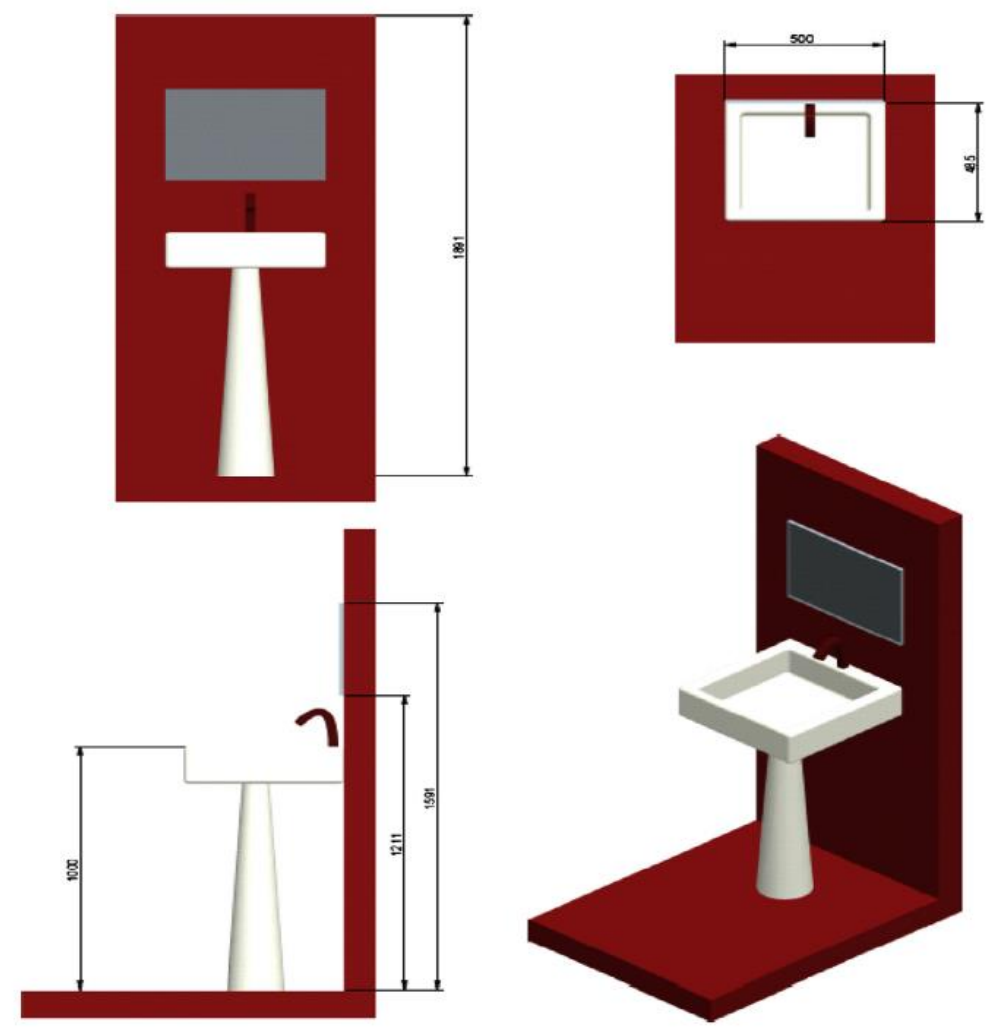

Fig. 5. 95th percentile male (all in $\mathrm{mm}$ ).

for designing bathrooms, lavatories, and kitchens based on the anthropometry of Malaysian elderly.

3.4. Recommended guidelines for designing basic bathrooms of the older Malaysian

The recommended guidelines for the bathroom areas included shower, toilet and hand basin area. The essential features for this area are wider doors; grab bars in shower and toilet, curbless shower, lever-style door handles and faucets, toilet with no-slam lids, wall-mounted sinks and wheelchair clearance for the disabled elderly. The bathroom design is shown in Figs. 3 and 4 . The dimensions of the bathroom area are $8 \mathrm{ft} 6 \mathrm{in} . \times 8 \mathrm{ft}$. This is to allow wheelchair clearance for the disabled elderly. The width for the shower area needs to cover the elbowroom including clearance which is 95th percentile male $(92 \mathrm{~cm}+$ $20 \mathrm{~cm}=112 \mathrm{~cm})$ and 5th female $(62.60 \mathrm{~cm}+20 \mathrm{~cm}=$ $82.60 \mathrm{~cm}$ ). The optimum height for the fixed showerhead should be sufficient to accommodate stature height including clearance. For 95th percentile male should cover $180 \mathrm{~cm}(170+10=180 \mathrm{~cm})$ and 5 th percentile female $145 \mathrm{~cm}(135 \mathrm{~cm}+10 \mathrm{~cm}$. A single water controller is located in line with the showerhead and its height is at the standing waist height. For 95th percentile male
$96.5 \mathrm{~cm}$ and 5th percentile female is $75.5 \mathrm{~cm}$. The vertical handrail is placed in the shower area for balancing while bathing with the minimum waist height for 95th female is $96.5 \mathrm{~cm}$ from the floor and 5th percentile female $75.5 \mathrm{~cm}$ from the floor. The design for hand basin, shower and toilet are shown in figure. The lavatories area included a toilet seat, handrails and tissue holder. The toilet seat height is similar to the chair seat height should cover popliteal height, which is discussed in Section 2 (B), 5th percentile female is $38.0 \mathrm{~cm}$ and 95th percentile male is $52.8 \mathrm{~cm}$. The water hose is located on the right-hand side and the single-lever water control is at the sitting elbow height for both percentiles. The maximum horizontal distance is calculated using the method proposed by [21] which is using Pythagoras' theorem. The designs for bathroom, toilet and hand basin are shown in Figs. 2-7 below.

\subsection{Recommended guidelines for designing basic kitchen for the older Malaysian}

To design of cabinet kitchen for the elderly should consider the kitchen space, the features at the cabinet and the height and size of the cabinet drawer. These considerations are needed to make sure the elderly's activities in kitchen are made easier. The essential features for this area include

Link to Full-Text Articles :

http://www.sciencedirect.com/science/article/pii/S0263224114004102 